\title{
Comparing the Efficacy of Shear Bond Strength with Composite Attachments and Different Ceramic Surfaces-Zirconia, Lithium Disilicate, and Feldspathic Porcelain
}

\author{
Michael Backstrand*, Neira Algazzaz and Fusun Ozer \\ Department of Preventive and Restorative Sciences, School of Dental Medicine, University of Pennsylvania, Philadelphia, Pennsylvania, USA
}

*Corresponding author: Michael Backstrand, School of Dental Medicine, University of Pennsylvania, 240 South $40^{\text {th }}$ Street, Philadelphia, PA 19104, USA.
Received Date: May 31, 2021

Published Date: June 11, 2021

\begin{abstract}
Introduction: Dental practitioners utilize many different dental materials to fabricate durable and aesthetic fixed prostheses. The practitioners may not be considering the future treatment options of their patients. In the field of orthodontics, there have traditionally been concerns about the bond strength between brackets and ceramic crown materials. However, as the popularity of esthetic treatment options continues to grow, there will be increased importance in extending this bonding knowledge to the application of the composite buttons utilized in clear aligner therapies. Since this bonding performance with the crown materials has been shown to be an issue in dentistry today, we investigated the most retentive ceramicbased crown materials to deliver optimal composite shear bond strength for attachment buttons.

Methods: A search was performed on shear bond strength of composite resin materials to three common dental fixed prosthetic materials (zirconia, lithium disilicate, and feldspathic porcelain) from November 1997 to August 2020. The research study primer application, thermocycling controlled variables, and composite resin choice was assessed.

Results: The collective studies presented contradicting results due to the lack of controlled variables. An assessment was made based on the mean shear bond strengths of the several assessments conducted on each dental material. Using this knowledge, zirconia presented a slightly higher shear bond strength than the other two materials before applying thermocycling treatment; however, some studies with differing controlled variables contradict these findings.

Conclusion: Without conducting a direct research study with controlled variables comparing the three materials-with the same primer setup, thermocycling protocol, composite resin, and ceramic materials-it is difficult to compare the efficacy of their individual shear bond strengths. With the current knowledge assessed from the included literature, we can assume that zirconia has the highest shear bond strength before thermocycling, while lithium disilicate is strongest when using the thermocycling assessment.
\end{abstract}

\section{Introduction}

Orthodontic treatment is not only used to improve aesthetics but is also utilized to treat functional problems for the patient, including temporomandibular joint disfunction, malocclusion, and overjet/ overbite [1]. Many patients who require orthodontic treatment may also have fixed prostheses that impede the effectiveness of bonding orthodontic brackets and Invisalign composite buttons. Many adult patients may have received crown restorations before their orthodontic treatment choice, however, there have always been complications with composite resin bonding to existing crowns in the past [2]. Therefore, this literature review aims to determine the efficacy of the bond strength of Invisalign clear aligner composite buttons to the different types of ceramic crown materials; including 
zirconia, lithium disilicate, and feldspathic porcelain surfaces. We assume that since zirconia is the state-of-the-art material currently used in restorative dentistry today, it will maintain significant positive results in regard to bonding with composite resin compared to the other materials tested.

If, however, the other ceramic materials provide superior shear bond strength to the composite buttons in comparison with zirconia, they should be more heavily implemented in the treatment plans of patients who may need to get specific orthodontic corrections. Once these ceramic crown materials have been tested, restorative dentists will feel more confident using the most effective fixed prosthesis for a patient planning to undergo orthodontic treatment in the near future. Zirconia is used in many different procedures including implants, crowns, and bridges, mostly due to its remarkable toughness, radiopacity, and biocompatibility properties $[3,4]$. In its increasing popularity in dentistry, there have been complications with patients who have zirconia crowns attempting to undergo orthodontic treatment that "has offered a great challenge to dental zirconia research and development" [5]. This has prompted investigation into the clinical success of adhesion to several other materials. Zirconia is typically indicated as a core for restorations due to the high opacity it presents so that a proper aesthetic appearance can be achieved.

Lithium disilicate is another highly aesthetic, popular dental crown material used today and is utilized similarly to zirconia. The dental ceramic is highly regarded for its flexural strength, high fracture toughness, and easily adjustable translucency which implements the versatile material in many different procedures including veneers, inlays, onlays, and crowns. Lithium disilicate has been attributed as a ceramic material that specializes in "dissimilar material combinations" and it offers a unique solution for restoration fabrication [6]. Even though they are known to have significant flexural strength and fracture resistance, they are still prone to tensile strains which can be involved chewing. It can also be used in every area of the mouth, which does not hold true for all dental materials.

Feldspathic porcelain is a commonly used dental material for fabrication of laminate veneers today. It has been referred to as the "premier esthetic material", specifically for custom veneer restorations. However, compared to other materials, it is not the leading material in terms of functionality [7]. It is a highly aesthetic material with adjustable translucency that closely resembles the color and texture of natural enamel. This feature can be credited to the nature of feldspathic porcelains, "leucite-based glass-matrix dental ceramics" [8]. In fact, porcelains infused with highest levels of glass produce impressive esthetic quality considering that a lower makeup of filler particles will truly enhance the translucent properties. However, this reduction in filler particles also gives rise to less desirable mechanical properties including flexural strength and fracture toughness, particularly when it's compared to its counterpart, lithium disilicate [8]. Further, feldspathic porcelains exhibit brittleness and, therefore, are susceptible to occlusal forces. Interestingly, porcelains are second to dental caries for being the most common cause for restoration replacement after experiencing fractures [9]. There are several factors that may severely impact the shear bond strength that one must be mindful about. These include occlusal forces, poor design and preparation, laboratory errors, and physical trauma that can compromise the cohesive forces required of the feldspathic porcelain [10]. This review paper is expected potentially contribute to the field of orthodontics and expand the arsenal of restorative materials that maintain their effectiveness with orthodontic brackets and Invisalign treatment applications.

\section{Materials and Methods}

\section{Inclusion and exclusion criteria}

All articles referring to zirconia, lithium disilicate, and feldspathic porcelain from 1997 to 2020 were considered when deciding which to include in the review. In order to compare the shear bond strengths of composite resin materials to different ceramics surfaces, the studies with significant mean shear bond strength's (measured in MegaPascals, MPa) were included in this review. Articles testing these materials for measurements other than shear bond strength were not included in the literature review. Tests that were not specifically for shear bond strength were excluded from revie such as the Vicker's hardness test, flexural strength, and more. Additionally, studies that focused their research on different surface conditioning methods to evaluate shear bond strength were included in the evaluation only if the study provided an accurate comparison of the mean shear bond strengths. This also holds true within the context of comparing different composite resins; papers are only included if the average of the shear bond strengths can be determined. Further, literature that was heavily focused on comparing the surface treatments as opposed to the shear bond strength of the material was excluded. After reviewing all papers with the given key phrase, 26 final papers were used for the analysis of this literature review.

\section{Search methodology}

The search methodology for this literature review was done through the PubMed database by using key phrase "Shear Bond Strength of Composite Resins to Ceramic Surfaces" regarding zirconia, lithium disilicate, feldspathic porcelain, shear bond strength, ceramic, composite resin, orthodontic treatment, and clear aligners.

\section{Search strategy}

All pertaining papers along with their reference lists were reviewed to be under consideration for potential inclusion. The precise phrase used in titles or abstract searches within the PubMed.gov database is "Shear Bond Strength of Composite Resins to Ceramic Surfaces." Papers published from November 1997 to November 2020 were considered for review. Of the 298 papers retrieved from the search strategy, 268 failed to meet the 
inclusion criteria. In particular, 148 papers were omitted due to meeting the exclusion criteria of studies focusing on surface treatment, 97 papers were outside the scope of objectives, and 23 due to meeting the exclusion criteria of orthodontic brackets. There were no duplicate papers to be omitted. Hence, 26 papers were accepted and identified key information that gives insight into the nature of the researched dental prostheses. Of these 26 papers, 16 papers contributed to quantitative discussion. Please refer to Table1- Criteria for Literature Review and Figure 1- PRISMA flowchart of Study Inclusion and Exclusion Criteria for supplemental information (Table 1) (Figure 1).
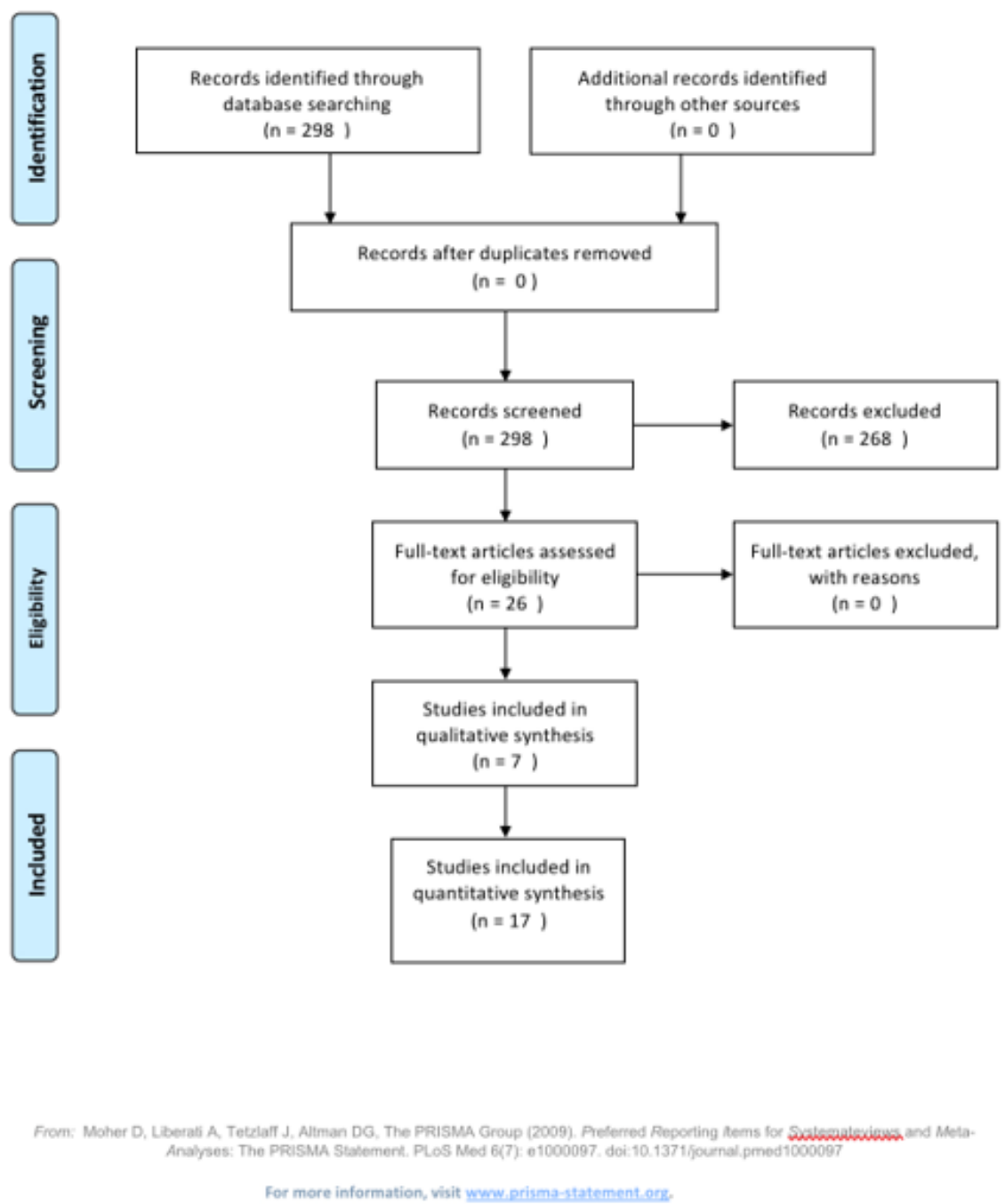

Figure 1: PRISMA flow-chart of Study Inclusion and Exclusion Criteria.

Table 1: Criteria for Literature Review

\begin{tabular}{|c|c|}
\hline Source & Criteria \\
\hline Database: & PubMed, Dentistry \\
\hline Date of Publication: & $1997-2020$ \\
\hline Key phrase: & "Shear Bond Strength of Composite Resins to Ceramic Surfaces" \\
\hline Language: & English \\
\hline Type of Paper: & In vitro laboratory studies \\
\hline Inclusion Criteria: & Studies focusing on shear bond strength of composite resin onto zirconia, lithium disilicate, or feldspathic porcelain \\
\hline Exclusion Criteria: & In vivo studies, orthodontic brackets, dental protheses not listed above, strength tests that are not shear bond strength, \\
studies focusing on surface treatment rather than shear bond strength
\end{tabular}


*Of the 274 failed to meet the inclusion criteria, 148 papers were omitted due to meeting the exclusion criteria of studies focusing on surface treatment, 97 papers were outside the scope of objectives, and 23 due to meeting the exclusion criteria of orthodontic brackets (Table 2).

Table2: Experimental Studies of Derived Shear Bond Strength Values.

\begin{tabular}{|c|c|c|c|c|c|}
\hline Paper & Author & Type of Study & n & Material & Shear Bond Strength Results (SBS in MPa) \\
\hline 2 & Juntavee et al. (2018) & In vitro & 15 & $\begin{array}{l}\text { Feldspathic por- } \\
\text { celain }\end{array}$ & $\begin{array}{c}\text { Mean SBS: } 12.65,14.50,13.97,12.40,15.85,14.06,12.12,15.65 \text {, } \\
12.89\end{array}$ \\
\hline 3 & Ruys (2015) & In vitro & 43 & Zirconia & 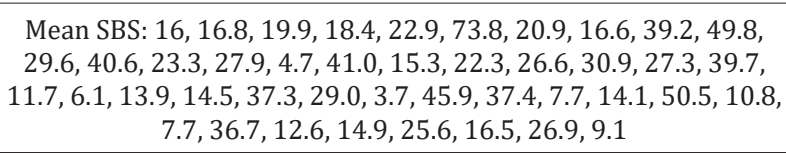 \\
\hline 9 & $\begin{array}{l}\text { Kukiattrakoon, et al. } \\
\text { (2012) }\end{array}$ & In vitro & 156 & $\begin{array}{l}\text { Feldspathic por- } \\
\text { celain }\end{array}$ & Highest SBS: 18.0 \\
\hline 10 & $\begin{array}{l}\text { Mohammadi, et al. } \\
\text { (2015) }\end{array}$ & In vitro & 156 & $\begin{array}{l}\text { Feldspathic por- } \\
\text { celain }\end{array}$ & Mean SBS: 19.1, 10.48, 21.33, 17.64, 11.63, 19.78 \\
\hline 11 & Kansal, et al. (2018) & In vitro & 56 & $\begin{array}{l}\text { Lithium disilicate, } \\
\text { zirconia }\end{array}$ & Zirconia: 42.95 Lithium disilicate: 8.74 \\
\hline 12 & Upadhyaya (2019) & In vitro & 45 & Lithium disilicate & Mean SBS groups I-III: 14.19, 10.70, 5.46 \\
\hline 13 & $\begin{array}{l}\text { Matsumura, et al. } \\
\text { (1997) }\end{array}$ & In vitro & 30 & $\begin{array}{l}\text { Feldspathic por- } \\
\text { celain }\end{array}$ & SBS: 30.0 \\
\hline 14 & Kimura, et al. (2019) & In vitro & 22 & Zirconia & $\begin{array}{c}\text { Median SBS: } 9.5,10.6,14.2,0.7,4.6,11.0,13.8,34.0,22.6,0.8 \\
13.8,0.9\end{array}$ \\
\hline 15 & Sanal, et al. (2020) & In vitro & 96 & $\begin{array}{l}\text { Feldspathic por- } \\
\text { celain }\end{array}$ & Mean SBS: $2.3,5.0$ \\
\hline 16 & Abu Alhaija (2010) & In vitro & 48 & $\begin{array}{l}\text { Lithium disilicate, } \\
\text { feldspathic por- } \\
\text { celain }\end{array}$ & Lithium Disilicate: 88.08 Feldspathic porcelain: 99.80 \\
\hline 18 & Huang, et al. (2001) & In vitro & 64 & $\begin{array}{l}\text { Feldspathic por- } \\
\text { celain }\end{array}$ & $\begin{array}{l}\text { Orthodontic forces are able to be tolerated at a minimum of 6-8 } \\
\qquad \mathrm{MPa}\end{array}$ \\
\hline 19 & Zakavi, et al. (2019) & In vitro & 40 & Zirconia & Mean SBS groups I-IV: 16.87, 13.44, 11.65, 6.87 \\
\hline 21 & Kubochi, et al. (2017) & In vitro & 11 & Zirconia & Zirconia: 14.1 \\
\hline 23 & Cevik (2018) & In vitro & 60 & $\begin{array}{l}\text { Feldspathic por- } \\
\text { celain }\end{array}$ & Mean SBS groups I-VI: 8.58, 6.51, 3.37, 2.71, 1.17, 0.93 \\
\hline 24 & Naranjo, et al. (2015) & In vitro & 100 & Lithium disilicate & Lithium disilicate: 15.1 \\
\hline 25 & Martina, et al. (1997) & In vitro & 90 & Zirconia & $\begin{array}{c}\text { SBS of new samples: } 15.52 \text { SBS after one recycling process: } 11.23 \\
\text { SBS after five recycling processes: } 10.10 \text { SBS after ten recycling } \\
\text { processes: } 10.04\end{array}$ \\
\hline 26 & Graiff, et al. (2008) & In vitro & 40 & $\begin{array}{l}\text { Feldspathic por- } \\
\text { celain }\end{array}$ & $\begin{array}{l}\text { Feldspathic porcelain without salinization SBS: } 22 \text {. Feldspathic } \\
\text { porcelain with salinization SBS: } 29 \text {. Salinization increases the } \\
\text { SBS. }\end{array}$ \\
\hline
\end{tabular}

Abbreviations: SBS: Shear Bond Strength; MPa: MegaPascal

\section{Results}

The most comparable results from each material was found to be articles that compared either different composite resins or bonding agents for the ceramic. In this case, the evaluation was made by comparing the averages of many different tests on the same dental material. The average shear bond strength of zirconia using this method was found to be $12.21 \mathrm{MPa}$ [11]. Using this same evaluation for lithium disilicate and feldspathic porcelain, the mean shear bond strengths were 10.19 MPa and 3.88 MPa, respectively [12, 13]. This method shows that zirconia serves as a superior fixed prosthesis, when optimizing for shear bond strength. However, with the lack of direct research comparing the shear bond strengths of the common dental materials, it is hard to compare since most of the studies investigate different methods of pretreatment and thermocycling. To achieve proper results, a study must be conducted with the same preparation of the material, thermocycling, and composite resins. In the context of CAD/CAM-manufactured composite resin to zirconia, Kimura, et al. [14] confirmed their rejection of the null hypothesis that thermocycling does not impact shear bond strength. Specifically, the shear bond strength of zirconia specimens was significantly higher in the Katana Avencia Block 12/A2 LT as well as the group treated with $9.5 \% \mathrm{HF}$, when compared to the lithium disilicate glass ceramics, with a median shear bond strength of 11.0, 13.8 and 0.9 MPa respectively [14]. Interestingly, Sanal, et al. [15] observed that thermal aging reduced the values of shear bond strength by $2.3 \mathrm{MPa}$, specifically within the context of composite resin [15]. 
One study evaluated did directly compare the shear bond strength of zirconia and lithium disilicate [11]. This research was able to conduct a study using the same controlled variables. In this case, the zirconia again held superior to the lithium disilicate, before thermocycling. After the thermocycling treatment, however, the lithium disilicate had a significantly higher shear bond strength. Before thermocycling, the values for shear bond strength of zirconia compared to lithium disilicate were $164.28 \mathrm{MPa}$ and $120.62 \mathrm{MPa}$, respectively. Thermocycling both dental materials with the same primer treatment and same composite resin changed the results whereby the lithium disilicate had a higher shear bond strength value of $42.95 \mathrm{MPa}$, compared to the $8.72 \mathrm{MPa}$ of zirconia. When evaluating lithium disilicate alone with different composite resins, its average shear bond strength was less than that of zirconia when studied alone. This value was $10.11 \mathrm{MPa}$, roughly $2 \mathrm{MPa}$ lower than the zirconia average shear bond strength [11]

Abu Alhaija, et al. [16] further confirms that the shear bond strength of lithium disilicate was much higher when compared to that of feldspathic porcelain [16]. Although a single study compared the two dental materials with appropriate controlled variables, the evaluation should not be deemed significant without a direct study on the desired materials. We lack detailed knowledge on the shear bond strengths of these three common dental prostheses due to the lack of in vitro research studies conducted on this subject. Publications comparing dental materials and their shear bond strengths tend to focus on the surface treatment and the type of composite resin used rather than comparing the shear bond strengths of each dental material. It is important to note, however, that different techniques of placement of composite resin can impact the value of the shear bond strength. For instance, the stair-step chamfer technique was especially beneficial to produce desirable results, both in the context of esthetics and shear bond strength with regards to orthodontic treatment [17].

For this to be properly achieved, a bench work study must be conducted with the same surface treatment and composite resin, directly comparing this universal protocol with the different dental materials in question. Merely comparing different dental material shear bond strengths is arbitrary when each study compared does not follow identical protocol. In the same way, in vitro studies can hold major disadvantages that may invalidate their credibility. The many dimensions of the oral cavity are far too variable to rely on conclusions drawn within in vitro studies. These dimensions can include $\mathrm{pH}$ levels, temperature, humidity, occlusal stresses, and plaque [16].

\section{Discussion}

Dental ceramics exhibit variability in shear bond strength amongst one another. The role of the shear bond strength of a dental ceramic to a composite resin is important in the field of dentistry, specifically for orthodontics, as the presence of dental prostheses can impair the adhesion of composite resin. As cited by Huang, et al. [18] the orthodontic forces can be tolerated at a minimum of 6-8 MPa shear bond strength [18]. Taking into consideration those values, one can project how the shear bond strength values obtained from in vitro studies would reflect into clinical practice.

\section{Zirconia}

In order to evaluate the shear bond strength of zirconia to composite resin, a different variety of adhesives were implemented [19]. Using forty zirconia blocks $(10 \times 10 \times 5 \mathrm{~mm})$ the specimens were divided into 4 groups and provided their own respective bonding agents including Futurabond U, Clearfil Universal Bond, Z-Prime Plus, and a zirconia primer. Composite resin was then applied to each ( $5 \mathrm{~mm}$ diameter, $2 \mathrm{~mm}$ thick). After thermocycling between 5 degrees Celsius and 55 degrees Celsius for 5000 cycles the shear bond strength was then evaluated.

The mean shear bond strength of group I was $16.8740 \mathrm{MPa}$, 13.4434 MPa in group II, 11.6500 MPa in group III, and $6.8700 \mathrm{MPa}$ in group IV (which was significantly lower than the other groups) [19]. The average of these four groups was $12.2094 \mathrm{MPa}$.

The purpose of this study was to compare different bonding agents with zirconia to assess their shear bond strength in restorations since this veneer-tooth interface is commonly a reason for failure in restorations. Studies have shown that the composition of adhesive used can play a role in what is considered a "clinically acceptable shear bond force" [20]. The results showed on average that after 5000 rounds of thermocycling there was a significant decrease in shear bond strength of all bonding agents with zirconia. Based on these results, universal adhesives had the highest efficacy of shear bond strength with zirconia. This is a significant advantage since not only does it have superior strength compared to the other bonding agents tested, it also has a wide variety of dental materials it is compatible with. These high-quality bonds and eclectic compatible dental materials allow for an ease in procedure steps when applying the zirconia. In fact, changing the variables of pressure, time, and size of the air abrading particles will only impact and preserve long-term shear bond strength if performed after thermocycling. Otherwise, no significant impact was observed if performed prior to thermocycling.

Additionally, different dental materials involved can ultimately decide the shear bond strength zirconia presents. Kubochi, et al. [21] reported that the maximum shear bond strength in relation to zirconia significantly increased from 14.1 MPa to 30.2 MPa when bonded to composite material and acrylic resin, respectively [21]. It should be pointed out, however, that surface treatment also had a significant effect on the efficacy of each bonding agent and its shear bond strength with zirconia restorations.

Blatz, et al. [22] studied the success of bonding of zirconia in the long-term by taking into consideration the impact of thermal cycling as well as Airborne-Particle Abrasion (APA). When three different adhesive luting cements both with and without APA was used on 
composite resin was bonded to zirconia, results shows that the shear bond strength was reduced considerably in the presence of APA. Additionally, the shear bond strength consequently decreased after the materials were put under 20,000 cycles [22].

Today, the most popular techniques to improve mechanical retention and shear bond strength with zirconia specimens include APA with alumina particulars associated with an adhesive/cement system as well as APA with silica-coated alumina particles followed with a silane coupling agent. Findings presented with a consistently high SBS measurement under the mentioned clinical strategies, such as $73.8 \mathrm{MPa}$, when compared to its counterparts as low as 7.7 MPa under the treatment of APA and resin cement [3].

\section{Lithium Disilicate}

The results of a study comparing zirconia and lithium disilicate directly found zirconia had a better shear bond strength than lithium disilicate. This study compared the shear bond strength of zirconia with and without thermocycling alongside lithium disilicate with and without thermocycling. The thermocycling emulated temperature ranges similar to those in the oral cavity and exposed the materials to these ranges in order to judge the efficacy of the materials under in vitro conditions. This will account for the thermal expansion and contraction between the tooth structure and materials used. Although the article was able to prove that zirconia had a stronger shear bond strength, this was only held true when not exposed to thermocycling. The shear bond strength mean averages of lithium disilicate exposed to thermocycling and not exposed to thermocycling were $42.95 \mathrm{MPa}$ and $120.62 \mathrm{MPa}$, respectively. On the other hand, zirconia's most effective test values were 8.72 MPa and 164.28 MPa, respectively [11].

Another study comparing different composite resins and their shear bond strength on lithium disilicate was evaluated as well. Using 45 lithium disilicate discs ( $4 \mathrm{~mm}$ diameter, $3 \mathrm{~mm}$ thick), the shear bond strength was tested with an average of $10.118 \mathrm{MPa}$ when bonded to human dentin. Upadhyaya, et al. [12] learned that total etch resins were concluded to be reliable and used widely in the clinical setting due to its durability [12]. Interestingly, Naranjo, et al. [23] reported that a higher shear bond strength was observed in the bonding ceramic brackets to lithium disilicate when compared to enamel, providing promising clinical advantages [23].

When compared to zirconia, lithium disilicate has demonstrated less shear bond strength in an experiment conducted by Kansal, et al. [11] as 28 zirconia specimens and 28 lithium disilicate specimens were subjected to a universal testing machine. Specifically, the lithium disilicate specimens presented a mean shear bond strength of $42.95 \mathrm{MPa}$ while the zirconia specimens presented with a value of 120.62 MPa [8].

\section{Feldspathic Porcelain}

An article comparing different surface treatments for the application of composite resin onto feldspathic porcelain evaluated the mean shear bond strengths. A total of 60 feldspathic porcelain discs were separated into 6 different groups: $37 \%$ phosphoric acid (G-H3P04), 9.4\% hydrofluoric acid (G-HF), grinding with diamond burs (G-Grinding), Nd:YAG laser (G-Nd:YAG), APA (G-Abrasion), and untreated specimens (G-Untreated) [24]. Each of these specimens were subjected to 2500 rounds of thermocycling between 5 and 55 degrees Celsius. The range of the shear bond strength values was from $0.93 \mathrm{MPa}$ and $8.58 \mathrm{MPa}$ with a total average of 3.88 MPa.

An additional in vitro study comparing disc shaped feldspathic porcelain specimens were treated with different priming agents. The evaluation showed that all three preparations of priming materials with feldspathic porcelain "exhibited consistent shear bond strength" greater than $30 \mathrm{MPa}$ after 20,000 thermocycles [13].

Interestingly, a study conducted by Huang, et al. [18] discussed the nature of feldspathic porcelain in the context of orthodontic forces. Particularly, they noted that the occurrence of damaged porcelain consequently increased as the value of the mean shear bond strength was greater [18]. This can be credited to the fact that ceramic brackets are considerably brittle and, hence, a lower shear bond strength value is desired. The more a specimen experienced recycling, the lower the shear bond strength value was. Recycling is a technique that allows a used bracket to be rebounded to enamel by removing the adhesive from the bracket followed by polishing, eliminating the impurities related to orthodontic treatment. For instance, the values for new ceramic brackets, brackets recycled once, brackets recycled five times, and bracket recycled ten times displayed a mean shear bond strength of 15.52, 11.23, 10.10, and 10.04, respectively [25].

Additionally, salinization has been reported to impact the shear bond strength of feldspathic porcelain. Graiff et al. [26] observed an increase of $7 \mathrm{MPa}$ with salinization when tested on 40 cylinders of feldspathic porcelain blocks that were abraded with hydrofluoric acid [26]. Further, different bonding systems can increase the value of the shear bond strength, more so than the type of adhesive or the type of composite resin. In the context of feldspathic porcelain, laser-treatment has been shown to produce a more uniform treated surface despite not having the highest bond strength, and is, therefore, considered a desirable option. In fact, laser treatment presented with a SBS of $12.89 \mathrm{MPa}$, while its counterparts only demonstrated an insignificant increase by $1.7 \mathrm{MPa}$ [2].

In terms of fluoride gel etching time, the shear bond strength of 156 feldspathic specimens as Kukiattrakoon, et al. [9] was investigated. HF etching exhibited the highest SBS of 18.0 MPa. However, it is comparable to APF etching for 6-10 minutes which yielded 16.0 MPa [9]. To add, an article written by Mohammadi, et al. [10] investigated the effect of different composite resins (methacrylate-based and Silorane-based) on the shear bond strength of feldspathic porcelain. Remarkably, both composite resin systems demonstrated high shear bond strength values. However, the application of Silorane adhesive exhibited a mean 
shear bond strength of $21.33 \mathrm{MPa}$, whereby the value decreased to $10.48 \mathrm{MPa}$ when Clearfil SE bond was applied [10]. Considering that the above studies were performed with different protocols and bonding techniques, the comparison on the shear bond strength values should be taken with caution. Nevertheless, an indirect comparison, taking into account the differences, may be impactful in determining how the different materials exhibit their respective shear bond strengths in the variable conditions of the oral cavity.

\section{Conclusion}

After evaluating many papers, whether included or excluded from this literature review, the data of shear bond strength superiority between zirconia, lithium disilicate, and feldspathic porcelain is difficult to pinpoint without controlled variables. On the one hand, the literature review was effective because the papers that did employ the same protocol were used to compare the shear bond strengths of different materials. On the other hand, the literature review also included papers that displayed the shear bond strengths but with different protocols. Hence, said papers were unable to collect significant data for direct and effective comparison, as opposed to a bench work study whereby the shear bond strength values of the different materials can be investigated within controlled conditions.

\section{Acknowledgment}

The authors are grateful for Laurel Graham, Head of Penn Dental Medicine Library for guidance and organization for this systematic review. The research did not receive any specific grant from funding agencies in the public, commercial, or not-for-profit sectors.

\section{Conflict of Interest}

The authors have no conflict of interest to report.

\section{References}

1. Harrison Jayne (2011) Orthodontic Treatment. Nature: 31-35.

2. Juntavee Niwut (2018) Shear bond strength of ceramic bracket bonded to different surface-treated ceramic materials. J Clin Exp Dent 10(12): e1167-e1176.

3. Ruys, Andrew J (2015) Zirconia as a Dental Biomaterial.

4. Samran A, Al-Ammari A, El Bahra S, Halboub E, Wille S, et al. (2019) Bond strength durability of self-adhesive resin cements to zirconia ceramic: An in vitro study. J Prosthet Dent 121(3): 477-484.

5. Bona, Alvaro Della (2015) Zirconia as a Dental Biomaterial. Materials (Basel, Switzerland) 8(8): 4978-4991.

6. Tysowsky George (2009) The Science Behind Lithium Disilicate: A Metal-Free Alternative. Dentistry Today 28(3): 112-113.

7. (2011) Feldspathic veneers: what are their indications?. Compend Contin Educ Dent 32(3): 44-49.

8. Noda Y, Nakajima M, Takahashi M, Mamanee T, Hosaka K, et al. (2017) The effect of five kinds of surface treatment agents on the bond strength to various ceramics with thermocycle aging. Dent Mater J 36(6): 755761.
9. Kukiattrakoon B, Thammasitboon K (2012) Optimal acidulated phosphate fluoride gel etching time for surface treatment of feldspathic porcelain: on shear bond strength to resin composite. Eur J Dent 6(1): 63-69.

10. Mohammadi N, Shakur Shahabi M, Kimyai S, Pournagi Azar F, Ebrahimi Chaharom ME (2015) Shear Bond Strengths of Methacrylate- and Silorane-based Composite Resins to Feldspathic Porcelain using Different Adhesive Systems. J Dent Res Dent Clin Dent Prospects 9(3): 181-187.

11. Kansal R, Rani S, Kumar M, Kumar S, Issar G (2018) Comparative Evaluation of Shear Bond Strength of Newer Resin Cement (RelyX Ultimate and RelyX U200) to Lithium Disilicate and Zirconia Ceramics as Influenced by Thermocycling. Contemp Clin Dent 9(4): 601-606.

12. Upadhyaya V, Arora A, Singhal J, Kapur S, Sehgal M (2019) Comparative analysis of shear bond strength of lithium disilicate samples cemented using different resin cement systems: An in vitro study. J Indian Prosthodont Soc 19(3): 240-247.

13. Matsumura H, Kato H, Atsuta M (1997) Shear bond strength to feldspathic porcelain of two luting cements in combination with three surface treatments. J Prosthet Dent 78(5): 511-517.

14. Kimura F, Komine F, Kubochi K, Yagawa S (2019) Bond strength of CAD/ CAM-manufactured composite resin and ceramic veneers to a zirconia framework. J Oral Sci 61(2): 327-334.

15. Sanal FA, Kilinc H (2020) Evaluating Ceramic Repair Materials in Terms of Bond Strength and Color Stability. Int J Prosthodont 33(5): 536-545.

16. Abu Alhaija ES, Abu AlReesh IA, AlWahadni AM (2020) Factors affecting the shear bond strength of metal and ceramic brackets bonded to different ceramic surfaces. Eur J Orthod 32(3): 274-280.

17. Eid H (2002) Retention of composite resin restorations in class IV preparations. J Clin Pediatr Dent 26(3): 251-256.

18. Huang TH, Kao CT (2001) The shear bond strength of composite brackets on porcelain teeth. Eur J Orthod 23(4): 433-439.

19. Zakavi Faramarz (2019) Evaluation of shear bond strength of zirconia to composite resin using different adhesive systems. J Clin Exp Dent 11(3): e257-e263.

20. Bishara SE, Olsen M, Von Wald L (1997) Comparisons of shear bond strength of precoated and uncoated brackets. Am J Orthod Dentofacial Orthop 112(6): 617-621.

21. Kubochi K, Komine F, Fushiki R, Yagawa S, Mori S, et al. (2017) Shear bond strength of a denture base acrylic resin and gingiva-colored indirect composite material to zirconia ceramics. J Prosthodont Res 61(2): 149-157.

22. Blatz MB, Sadan A, Maltezos C, Blatz U, Mercante D, et al. (2004) In vitro durability of the resin bond to feldspathic ceramics. Am J Dent 17(3):169-172.

23. Naranjo J, Ali M, Belles D (2015) Comparison of shear bond strength of self-etch and self-adhesive cements bonded to lithium disilicate, enamel and dentin. Tex Dent J132(11): 914-921.

24. Cevik P, Eraslan O, Eser K, Tekeli S (2018) Shear bond strength of ceramic brackets bonded to surface-treated feldspathic porcelain after thermocycling. Int J Artif Organs 41(3):160-167.

25. Martina R, Laino A, Cacciafesta V, Cantiello P (1997) Recycling effects on ceramic brackets: a dimensional, weight and shear bond strength analysis. Eur J Orthod 19(6): 629-636.

26. Graiff L, Piovan C, Vigolo P, Mason PN (2008) Shear bond strength between feldspathic CAD/CAM ceramic and human dentine for two adhesive cements. J Prosthodont 17(4): 294-329. 